\title{
Quantitative assessment
} CrossMark of mesenchymal stem cells contained in concentrated autologous bone marrow aspirate transplantation for the treatment of osteonecrosis of the femoral head: predictive factors and differences by etiology

\author{
Hiroshi Kumagai ${ }^{1}$, Tomokazu Yoshioka ${ }^{1 *}$, Hisashi Sugaya ${ }^{1}$, Yohei Tomaru' ${ }^{1}$, Yukiyo Shimizu², Masashi Yamazaki ${ }^{1}$
} and Hajime Mishima ${ }^{1}$

\begin{abstract}
Objective: We previously established concentrated autologous bone marrow aspirate transplantation as a one-step, lowly invasive, joint-preserving surgical technique for treating osteonecrosis of the femoral head. The objectives of this study were to identify factors that may predict the mesenchymal stem cell (MSC) count in bone marrow aspirate, concentrated using our method, and to clarify etiology related differences in the number of MSCs in concentrated bone marrow aspirate.

Results: The MSC counts per $10^{6}$ nucleated cells before concentration in the steroid, alcohol, and trauma groups were $2.31 \pm 2.96,2.58 \pm 2.30$, and $1.95 \pm 1.85$, respectively. The MSC counts per $10^{6}$ nucleated cells after concentration were $3.23 \pm 3.41,3.30 \pm 2.83$, and $2.56 \pm 1.98$ cells, respectively. The MSC concentration rates in the steroid, alcohol, and trauma groups were $7.15 \pm 5.62,5.08 \pm 1.96$, and $8.23 \pm 4.82$ times, respectively. None of the differences were significant. Multiple regression analysis revealed that MSC count was related to the total bone marrow aspirated, peripheral blood platelet count, and nucleated cell count in the initial aspiration.
\end{abstract}

Keywords: Concentrated autologous bone marrow aspirate transplantation, Osteonecrosis of the femoral head, Joint-preserving surgery, Mesenchymal stem cells

\section{Introduction}

We developed a simple and efficient method for collecting mesenchymal stem cells (MSCs) by centrifuging bone marrow aspirate to concentrate and extract a buffcoat layer containing MSCs, which may be used to treat osteonecrosis of the femoral head (ONFH) $[1,2]$. The main characteristics of this procedure are that it takes

\footnotetext{
*Correspondence: tymd99@tsukuba-seikei.jp

${ }^{1}$ Department of Orthopaedic Surgery, Faculty of Medicine, University of Tsukuba, Tsukuba, Ibaraki, Japan

Full list of author information is available at the end of the article
}

approximately $2 \mathrm{~h}$ from bone marrow aspiration to transplantation into the necrosis site and the fact that it is a one-step joint-preserving surgery.

Muschler et al. [3] reported that progenitor cells constitute approximately 1 per 30,000 nucleated cells in an iliac bone marrow aspirate. Hernigou et al. $[4,5]$ reported positive outcomes in the treatment of ONFH and nonunion by concentrating close to 600 progenitor cells $/ \mathrm{mL}$ of iliac bone marrow aspirate to approximately 2500 cells/ $\mathrm{mL}$ using a cell separator. They also reported a formula for predicting the number of nucleated cells in bone marrow as follows: $N\left(10^{8} / \mathrm{kg}\right)=(V \times \mathrm{NP})-(V-100) \times \mathrm{NS} / P$, 
where $V$ is the total volume of aspirate, NP is the nuclear cell count per milliliter of bone marrow aspirate, NS is the nuclear cell count per $\mathrm{ml}$ of peripheral blood, and $P$ is the patient's weight [4].

Our method for concentrating bone marrow aspirate does not use a cell separator [1]. Previously, we reported positive outcomes gained by using this method in the treatment of ONFH $[2,6]$. It has also been applied in the treatment of nonunion [7].

In the present study, we cultured a portion of the bone marrow aspirate for transplantation to estimate MSC count on the basis of fibroblastic colony- forming unit (CFU-F). However, as we did not perform cell culture or predictive screenings, we could not calculate the MSC count in bone marrow aspirate during the procedure.

Furthermore, reviewed literatures did not indicate whether differences in MSC counts were based on etiology. Approximately half (51\%) of ONFH cases in Japan are steroid related [8]. Therefore, the effects of steroid therapy on the quality and quantity of bone marrow aspirates must be investigated. Predicting the number of MSCs prior to bone marrow transplantation may be useful for clarifying the efficacy and limitations of this procedure.

The objectives of this study were to identify predictive factors of and clarify etiology-related differences in MSC count in concentrated bone marrow aspirate.

\section{Main text \\ Materials and methods \\ Patients}

The study subjects were 93 patients (60 men and 33 women) who underwent joint-preserving surgery for ONFH at our hospital between November 2012 and May 2017. The etiology was steroid related in 58 patients, alcohol related in 18, and trauma related in 17 . In this series, of the 93 patients, 58 (62.4\%) had steroid-related ONFH and 22 (38.0\%) had steroid-related ONFH with systemic lupus erythematosus (SLE). Of the 32 men and 26 women, $6(18.8 \%)$ and $16(61.6 \%)$ had SLE, respectively. The mean age was $41.0 \pm 11.5$ years (Table 1 ).

\section{Bone marrow aspiration, concentration, and transplantation}

Bone marrow aspiration, concentration, and transplantation were performed using the method previously developed by Yoshioka et al. and Tomaru et al. [5, 6]. Firstly, bone marrow was aspirated from both anterior iliac crests using a bone marrow harvesting needle. The bone marrow aspirates were processed by a two-step centrifugation method at room temperature. The bone marrow concentrates containing buffy coat were extracted. This technique reduced the typical $300 \mathrm{~mL}$ of bone marrow
Table 1 Patient demographics

\begin{tabular}{ll}
\hline Number of enrolled patients & $\mathbf{9 3}$ \\
\hline Age range & $41.0 \pm 11.5$ \\
Male:female & $60: 33$ \\
Average BMI & $23.3 \pm 3.4$ \\
Etiology & \\
Steroid related & $58(\mathrm{M}: 32 \mathrm{~F}: 26)^{\mathrm{a}}$ \\
Alcohol related & $18(\mathrm{M}: 17 \mathrm{F:})$ \\
Trauma related & $17(\mathrm{M}: 11 \mathrm{~F}: 6)$ \\
\hline
\end{tabular}

a Steroid related patient with SLE 24 (M: 7 F: 17), without SLE 34 (M: 25 F: 9)

aspirate to bone marrow concentrates of approximately 30-40 mL.

Before transplantation, multidirectional holes are made to perforate the interface between the areas of ONFH by drilling with a Kirschner wire. Under bi-plane fluoroscopic control, the transplantation was performed. After the operation, weight bearing was limited for 6 weeks, while non-weight bearing exercise was allowed.

\section{Bone marrow aspirate evaluation}

During the operation, $2 \mathrm{~mL}$ of bone marrow aspirate containing anticoagulant citrate dextrose solution was collected from the bag, and another $2 \mathrm{~mL}$ was collected after being concentrated for culturing. Bone marrow aspirate samples were mixed with $8 \mathrm{ml}$ of phosphate-buffered saline (PBS) and centrifuged, and $9 \mathrm{~mL}$ of the supernatant was removed. Next, $4 \mathrm{~mL}$ of culture medium was added and $500-\mu \mathrm{L}$ portions of a $5-\mathrm{mL}$ total were seeded into 6-well dishes.

Each dish was seeded with $100 \mu \mathrm{L}$ of bone marrow aspirate, and each plate was seeded with $600 \mu \mathrm{L}$ of bone marrow aspirate, which was cultured at $37{ }^{\circ} \mathrm{C}$ in $5 \%$ carbon dioxide. The culture medium comprised of Dulbecco's modified Eagle's medium (Sigma, St. Louis, MO, USA), 10\% fetal bovine serum (Gibco, Grand Island, NY, USA), and 1\% antibiotic-antimycotic solution (Gibco). Each plate was filled with $2 \mathrm{~mL}$ of the medium, and the first medium replacement was performed $24 \mathrm{~h}$ later. Thereafter, the medium was replaced every 2-3 days, and crystal-violet staining was performed when the colonies could not be distinguished because of overlapping, or 14 days after seeding. Washing was conducted twice with PBS, and $2 \mathrm{ml}$ of crystal violet was added to each dish. The mixture was left to stand for $5 \mathrm{~min}$. The specimens were then rinsed with water, dried, and examined with a microscope. Colonies with diameters of $\geq 2 \mathrm{~mm}$ were counted to obtain the number of CFU-F. The mean of two counts was used as the measurement. 
For this study, MSC count was defined as the CFU-F count. Total bone marrow aspirated, nucleated cell count (peripheral blood and initial aspiration in bag after concentration), red blood cell (RBC) count (in bag after concentration), platelet count (in bag after concentration), and MSC count (in bag after concentration) were measured. Rates of concentration from the bone marrow aspirate in the bag to the concentrated bone marrow aspirate were calculated (nucleated cells, RBC, platelets and MSCs). We investigated whether the MSC count after concentration had sex-related differences. Among the patients with steroid-related ONFH, we compared the MSC count after concentration regardless of SLE.

Etiology-based differences in the specimens were statistically examined using the Student $t$ test. By using the concentrated MSC count as the dependent variable, multiple regression analysis was performed to determine factors (independent variables) that may predict the concentrated MSC count. The independent variables were age, body mass index, nucleated cell count (peripheral blood and initial aspiration in bag after concentration), RBC count (peripheral blood and initial aspiration after concentration), platelet count (peripheral blood and initial aspiration after concentration), peripheral blood fraction, nucleated cell concentration rate, RBC concentration rate, platelet concentration rate, and total bone marrow aspirated.

\section{Results}

The total bone marrow aspirated was $252.5 \pm 83.1 \mathrm{~mL}$ in the steroid group, $244.7 \pm 81.9 \mathrm{~mL}$ in the alcohol group, and $280.3 \pm 51.9 \mathrm{~mL}$ in the trauma group. The nucleated cell counts in the steroid, alcohol, and trauma groups in peripheral blood were $6.9 \pm 2.9 \times 10^{3} / \mu \mathrm{L}, 5.9 \pm 1.4 \times 10^{3} /$ $\mu \mathrm{L}$, and $6.0 \pm 2.1 \times 10^{3} / \mu \mathrm{L}$, respectively. The nucleated cell counts in the initial aspiration were $51.4 \pm 28.5 \times 10^{3} / \mu \mathrm{L}$, $53.2 \pm 25.6 \times 10^{3} / \mu \mathrm{L}$, and $46.4 \pm 22.9 \times 10^{3} / \mu \mathrm{L}$, respectively. The nucleated cell counts in the bag were $10.5 \pm 5.1 \times 10^{3}$ / $\mu \mathrm{L}, \quad 9.4 \pm 3.6 \times 10^{3} / \mu \mathrm{L}$, and $10.4 \pm 3.8 \times 10^{3} / \mu \mathrm{L}$, respectively. The nucleated cell counts after concentration were $40.3 \pm 23.6 \times 10^{3} / \mu \mathrm{L}, \quad 39.2 \pm 16.0 \times 10^{3} / \mu \mathrm{L}, \quad$ and $48.3 \pm 20.4 \times 10^{3} / \mu \mathrm{L}$, respectively.

The nucleated cell concentration rates in the steroid, alcohol, and trauma groups were $4.1 \pm 1.9,4.5 \pm 1.7$, and $4.8 \pm 1.9$ times, respectively. The $\mathrm{RBC}$ concentration rates were $1.2 \pm 0.5,1.0 \pm 0.3$, and $1.0 \pm 0.3$ times, respectively. The platelet concentration rates were $6.2 \pm 2.2,6.1 \pm 1.9$, and $7.5 \pm 2.5$ times, respectively.

The MSC counts per $10^{6}$ nucleated cells before concentration in the steroid, alcohol, and trauma groups were $2.31 \pm 2.96,2.58 \pm 2.30$, and $1.95 \pm 1.85$ cells, respectively. The MSC counts per $10^{6}$ nucleated cells after concentration were $3.23 \pm 3.41,3.30 \pm 2.83$, and $2.56 \pm 1.98$ cells, respectively.

Per milliliter of blood marrow aspirate, the counts before concentration in the steroid, alcohol, and trauma groups were $23.09 \pm 28.22,23.94 \pm 19.31$, and $19.22 \pm 21.74$ cells, respectively. The counts after concentration were $137.41 \pm 160.06,129.04 \pm 113.95$, and $103.99 \pm 54.71$ cells, respectively. The MSC concentration rates in the steroid, alcohol, and trauma groups were $7.15 \pm 5.62, \quad 5.08 \pm 1.96$, and $8.23 \pm 4.82$ times, respectively. None of these differences were significant (Table 2).

The mean MSC counts after concentration in the men $(151.3 \pm 146.6$ cells $)$ was higher than that in the women $(89.4 \pm 108.8$ cells) with a significant difference $(p<0.025)$. The mean MSC count after concentration in

Table 2 Cell count according to etiology

\begin{tabular}{|c|c|c|c|}
\hline & Corticosteroid treatment & Alcohol abuse & Trauma \\
\hline Total bone marrow aspirated $(\mathrm{mL})$ & $252.5 \pm 83.1$ & $244.7 \pm 81.9$ & $280.3 \pm 52.0$ \\
\hline Nucleated cell count: peripheral blood (× 1000/ $\mathrm{LL})$ & $6.9 \pm 2.9$ & $5.9 \pm 1.4$ & $6.0 \pm 2.1$ \\
\hline Nucleated cell count: initial aspiration $(\times 1000 / \mu \mathrm{L})$ & $51.4 \pm 28.5$ & $53.2 \pm 25.6$ & $46.4 \pm 22.9$ \\
\hline Nucleated cell count: before concentration ( $\times 1000 / \mu \mathrm{L})$ & $10.5 \pm 5.1$ & $9.4 \pm 3.6$ & $10.4 \pm 3.8$ \\
\hline Nucleated cell count: after concentration (× 1000/uL) & $40.3 \pm 23.6$ & $39.2 \pm 16.0$ & $48.3 \pm 20.4$ \\
\hline Nucleated cell count concentration rate (times) & $4.1 \pm 1.9$ & $4.5 \pm 1.7$ & $4.8 \pm 1.9$ \\
\hline Red blood cell concentration rate (times) & $1.2 \pm 0.5$ & $1.0 \pm 0.3$ & $1.0 \pm 0.3$ \\
\hline Platelet concentration rate (times) & $6.2 \pm 2.2$ & $6.1 \pm 1.9$ & $7.5 \pm 2.5$ \\
\hline MSC count: before concentration (per $10^{6}$ nucleated cells) & $2.3 \pm 3.0$ & $2.6 \pm 2.3$ & $2.0 \pm 1.9$ \\
\hline MSC count: after concentration (per $10^{6}$ nucleated cells) & $3.2 \pm 3.4$ & $3.3 \pm 2.8$ & $2.6 \pm 2.0$ \\
\hline MSC count: before concentration (per $1 \mathrm{~mL}$ bone marrow aspirate) & $23.1 \pm 28.2$ & $23.9 \pm 19.3$ & $19.2 \pm 21.7$ \\
\hline MSC count: after concentration (per 1 mL bone marrow aspirate) & $137.4 \pm 160.1$ & $129.0 \pm 114.0$ & $104.0 \pm 54.7$ \\
\hline MSC concentration rate (times) & $7.2 \pm 5.6$ & $5.1 \pm 2.0$ & $8.2 \pm 4.8$ \\
\hline
\end{tabular}

None of these differences were significant 
the patients with steroid-related ONFH patients with SLE (95.3 \pm 97.3 cells) was lower than that in the patients with steroid-related ONFH without SLE (164.7 \pm 95.3 cells; $p=0.114$ ). Multiple regression analysis indicated that the MSC count was related to the total bone marrow aspirated, peripheral blood platelet count, and nucleated cell count in the initial aspiration (Table 3 ).

\section{Discussion}

Transplantation of concentrated bone marrow aspirate for ONFH was first reported by Hernigou et al. [9]. Since 2003, our department has used a simplified method for concentrating bone marrow aspirate [1] and a one-step surgical procedure involving transplanting into the area of necrosis (concentrated autologous bone marrow aspirate transplants).

In the present study, etiology-based differences in MSC count were not observed. Kato et al. [10] examined the osteogenic differentiation capacity of adipose tissuederived MSCs in patients with steroid-induced ONFH and reported that steroid therapy may increase Dkk-1 expression level, which may reduce osteogenic differentiation capacity. Recent studies indicate that microRNA expression may affect osteogenic differentiation capacity [11, 12]. Chen et al. [13] reported that miR-708 may markedly suppress osteogenic and adipogenic differentiations of MSCs. On the other hand, the mean MSC count after concentration in the men was higher than that in the women. Jones et al. [14] indicated no significant difference in MSC count per milliliter of bone marrow aspirate between age-matched male and female subjects. In this study, a large difference was observed in the proportion of patients with steroid-related ONFH with SLE depending on their sex. Although no significant difference was found, the mean MSC count after concentration in the patients with steroid-related ONFH with SLE was lower than that in those with steroid-related ONFH without SLE. The relationship between SLE and MSC count is unknown. Caplan reported that autologous MSCs appeared to exhibit anti-inflammatory and immunomodulatory effects after transplantation for SLE [15].
It will also be important in the future to elucidate the links between SLE and MSC count.

Furthermore, the predictive factors assessed in the present study were total bone marrow aspirated, peripheral blood platelet count, and nucleated cell count in the initial aspiration. Hernigou et al. [4] reported that total bone marrow aspirated, nucleated cell count per milliliter of bone marrow aspirate, nucleated cell count per milliliter of peripheral blood, and body weight were factors that may predict the number of nucleated cells in bone marrow. These findings differ slightly from ours, which may be due to differences in the collection method for MSCs, as a cell separator was used by Hernigou et al.

If it were possible to predict the MSC count using preoperative test findings, it may be helpful in deciding on the type of surgical intervention needed. However, our study indicates that such a prediction would be difficult without resorting to bone marrow aspiration.

Some patients in the present study exhibited relatively low MSC counts. Examining individual cases and continuing to search for predictive factors may be important in achieving stable cellular therapies.

In conclusion, the post-concentration MSC count was related to the total bone marrow aspirated, peripheral blood platelet count, and nucleated cell count in the initial aspiration. Significant etiology-based differences were not observed in the MSC counts after concentration or MSC concentration rates, indicating that this collection method may be useful in the treatment of ONFH.

\section{Limitation}

A limitation of the present study was that while the blood count and CFU-F were measured, differentiation capacity, proliferation capacity, or other cellular characteristics were not evaluated. We did not perform flow cytometry analysis for all the patients, only in some patients, and cited previous literatures to describe our method [16].

Moreover, as only patients with ONFH were included in the study, whether similar results may be observed in heavy steroid users who do not exhibit ONFH remains

Table 3 Multiple regression analysis indicated that the MSC count

\begin{tabular}{lcccc}
\hline & $\begin{array}{l}\text { Unstandardized } \\
\text { coefficient }\end{array}$ & Standard error & $\begin{array}{c}\text { Standardized } \\
\text { coefficient } \boldsymbol{\beta}\end{array}$ & $\begin{array}{c}\text { t value value } \\
\text { statistics tolerance }\end{array}$ \\
\hline (Constant) & -164.486 & 42.669 & 0.35 & 0 \\
Total bone marrow aspirated & 0.475 & 0.104 & 0.426 & 0 \\
Platelet count: peripheral blood & 0.4 & 0.138 & 0.267 & 0.563 \\
$\begin{array}{l}\text { Nucleated cell count: initial bone } \\
\text { marrow aspiration }\end{array}$ & 0.617 & 0.303 & 0.191 & 2.897 \\
\hline
\end{tabular}


unclear. Therefore, it may be useful to evaluate mid- and long-term clinical outcomes based on etiology.

\section{Abbreviations}

ONFH: osteonecrosis of the femoral head; MSC: mesenchymal stem cell; CFUF: fibroblastic colony-forming unit; SLE: systemic lupus erythematosus; PBS: phosphate-buffered saline; RBC: red blood cell.

\section{Authors' contributions}

The study was planned and coordinated by HK, TY, HM, and MY. The data collection, analysis, and interpretation, and manuscript writing were performed by HK, HS, YT, YS, and TY. All authors read and approved the final manuscript.

\section{Author details}

${ }^{1}$ Department of Orthopaedic Surgery, Faculty of Medicine, University of Tsukuba, Tsukuba, Ibaraki, Japan. ${ }^{2}$ Department of Rehabilitation Medicine, Faculty of Medicine, University of Tsukuba, Tsukuba, Ibaraki, Japan.

\section{Acknowledgements}

We thank Editage (www.editage.jp) for English language editing

\section{Competing interests}

The authors declare that they have no competing interests.

\section{Availability of data and materials}

The datasets analyzed during the present study are available from the corresponding author upon reasonable request.

\section{Consent to publish}

Not applicable.

\section{Ethics approval and consent to participate}

All procedures were approved by an Institutional Ethics Review Committee of the University of Tsukuba. Written informed consent was obtained from all individuals who participated in this study prior to the operation.

\section{Funding}

There is no funding source.

\section{Publisher's Note}

Springer Nature remains neutral with regard to jurisdictional claims in published maps and institutional affiliations.

Received: 11 October 2018 Accepted: 23 November 2018 Published online: 29 November 2018

\section{References}

1. Sakai S, Mishima H, Ishii T, Akaogi H, Yoshioka T, Uemura T, et al. Concentration of bone marrow aspirate for osteogenic repair using simple centrifugal methods. Acta Orthop. 2008;79(3):445-8.
2. Yoshioka T, Mishima H, Akaogi H, Sakai S, Li M, Ochiai N. Concentrated autologous bone marrow aspirate transplantation treatment for corticosteroid-induced osteonecrosis of the femoral head in systemic lupus erythematosus. Int Orthop. 2011;35(6):823-9.

3. Muschler GF, Boehm C, Easley K. Aspiration to obtain osteoblast progenitor cells from human bone marrow: the influence of aspiration volume. J Bone Joint Surg Am. 1997;79(11):1699-709.

4. Hernigou P, Poignard A, Manicom O, Mathieu G, Rouard $\mathrm{H}$. The use of percutaneous autologous bone marrow transplantation in nonunion and avascular necrosis of bone. J Bone Joint Surg Br. 2005;87b(7):896-902.

5. Hernigou P, Poignard A, Beaujean F, Rouard H. Percutaneous autologous bone-marrow grafting for nonunions. Influence of the number and concentration of progenitor cells. J Bone Joint Surg Am. 2005;87(7):1430-7.

6. Tomaru Y, Yoshioka T, Sugaya H, Aoto K, Wada H, Akaogi H, et al. Hip preserving surgery with concentrated autologous bone marrow aspirate transplantation for the treatment of asymptomatic osteonecrosis of the femoral head: retrospective review of clinical and radiological outcomes at 6 years postoperatively. BMC Musculoskelet Disord. 2017;18(1):292.

7. Sugaya H, Mishima H, Aoto K, Li M, Shimizu Y, Yoshioka T, et al. Percutaneous autologous concentrated bone marrow grafting in the treatment for nonunion. Eur J Orthop Surg Traumatol. 2014;24(5):671-8.

8. Kubo T, Ueshima K, Saito M, Ishida M, Arai Y, Fujiwara H. Clinical and basic research on steroid-induced osteonecrosis of the femoral head in Japan. J Orthop Sci. 2016;21(4):407-13.

9. Hernigou P. Treatment of osteonecrosis with autologous bone marrow grafting. Clin Orthop Relat Res. 2002;405:14-23.

10. Kato T, Khanh VC, Sato K, Kimura K, Yamashita T, Sugaya H, et al. Elevated expression of Dkk-1 by glucocorticoid treatment impairs bone regenerative capacity of adipose tissue-derived mesenchymal stem cells. Stem Cells Dev. 2018;27(2):85-99.

11. Bian Y, Qian W, Li H, Zhao RC, Shan WX, Weng X. Pathogenesis of glucocorticoid-induced avascular necrosis: a microarray analysis of gene expression in vitro Int J Mol Med. 2015;36(3):678-84.

12. Li T, Li H, Li T, Fan J, Zhao RC, Weng X. MicroRNA expression profile of dexamethasone-induced human bone marrow-derived mesenchymal stem cells during osteogenic differentiation. J Cell Biochem. 2014;115(10):1683-91.

13. Hao C, Yang S, Xu W, Shen JK, Ye S, Liu X, et al. MiR-708 promotes steroidinduced osteonecrosis of femoral head, suppresses osteogenic differentiation by targeting SMAD3. Sci Rep. 2016;6:22599.

14. Jones $E$, Schafer R. Where is the common ground between bone marrow mesenchymal stem/stromal cells from different donors and species? Stem Cell Res Ther. 2015;6:143

15. Caplan Al. Why are MSCs therapeutic? New data: new insight. J Pathol. 2009;217:318-24

16. Sugaya H, Yoshioka T, Kato T, Taniguchi Y, Kumagai H, Hyodo K, et al. Comparative analysis of cellular and growth factor composition in bone marrow aspirate concentrate and platelet-rich plasma. Bone Marrow Res. 2018;2018:1-9.

Ready to submit your research? Choose BMC and benefit from:

- fast, convenient online submission

- thorough peer review by experienced researchers in your field

- rapid publication on acceptance

- support for research data, including large and complex data types

- gold Open Access which fosters wider collaboration and increased citations

- maximum visibility for your research: over 100M website views per year

At BMC, research is always in progress.

Learn more biomedcentral.com/submissions 